\title{
An Uncommon Cause of Bronchiectasis: A Case Report
}

\author{
UI Qadir $\mathbf{A}^{\mathbf{1}^{*}}$, Afsar $\mathbf{A}^{2}$ and Aslam $\mathbf{M}^{2}$
}

${ }^{1}$ Department of PUImonary Medicine, POF Hospital, Wah Medical College, Wah Cantt, Pakistan

${ }^{2}$ Department of Internal Medicine, POF Hospital, Wah Medical College, Wah Cantt, Pakistan

\begin{abstract}
Bronchiectasis is a common respiratory condition and has a number of causes. One of the causes of bronchiectasis is immunoglobUlin deficiency. Common variable immunodeficiency (CVID) is the most common symptomatic primary immunodeficiency disorder characterized by an increased incidence of recurrent infections, autoimmune phenomena and neoplastic diseases. This article describes a 37-year-old gentleman who was diagnosed as a case of CVID on the basis of recurrent respiratory tract infections and laboratory findings. He improved with monthly intravenous immunoglobUlins (IVIG). The diagnosis is often difficUlt and delayed but shoUld be considered for patients with recurrent infections.
\end{abstract}

Keywords: Bronchiectasis; CVID; HypoimmunoglobUIinemia; IVIG

\section{Introduction}

CVID is a primary immunodeficiency disorder [1] that involves low levels of most or all of the immunoglobUIin classes, lack of B lymphocytes and frequent bacterial infections [2]. Increased susceptibility to infections with encapsUIated organisms is the hallmark of this condition. Recurrent sinusitis, bronchitis, otitis, pharyngitis and pneumonia are commonly encountered infections. About $20 \%$ of patients present with gastrointestinal infections and sprue-like syndromes. The prevalence of CVID is about 1 in 25000 in the United States and one case per 50,000 popUIation worldwide [3]. CVID manifests itself between ages of 5-10 years and 20-40 years [4]. Genetics of CVID is still unknown. Demonstration of functional or qualitative defects in antibody production is essential and all patients have reduced levels of ImmunoglobUIin G (IgG), ImmunoglobUIin (IgA) and/or ImmunoglobUlin (IgM) [5]. Most incidences of pneumonia occur before the diagnosis of CVID and hence resUIt in sequelae like bronchiectasis, lung volume loss and empyema. Paradoxically there is increased incidence of autoimmune diseases (20\%). Treatment includes lifelong immunoglobUlin administration by subcutaneous (SC) or intravenous (IV) route. Here we report case of a 37-year-old gentleman with bronchiectasis secondary to recurrent infections of respiratory tract who was diagnosed to have CVID.

\section{Case Report}

A 37-year-old gentleman presented with history of recurrent lower respiratory tract infections since 2001 and was diagnosed as a case of bronchiectasis. In primary care setting he was treated with repeated courses of antibiotics but was never worked up appropriately for any underlying cause of his bronchiectasis. He was fertile. In 2003, he was started on anti-tubercUIosis therapy (ATT) empirically based on history of chronic productive cough and radiological findings. Patient did not recall whether his smear was positive or not. He took ATT for 3 months which was then stopped because his symptoms did not improve and there was no definitive evidence of Mycobacterium TubercUIosis (MTB) infection. In 2008 his condition deteriorated and he was hospitalized. He had developed left-sided empyema thoracis for which he was referred to thoracic surgeon. He underwent videoassisted thoracoscopic surgery (VATS) and decortication was done for empyema. He continued to have recurrent chest infections for which he had to take antibiotics (Figure 1).

He underwent left lower lobectomy in January 2014. Initially he showed improvement but then he was lost to follow-up. He

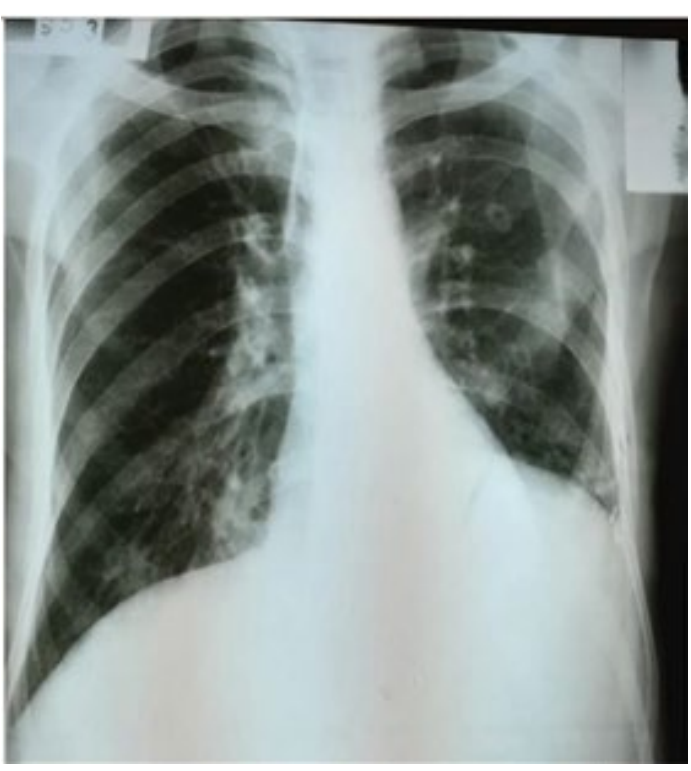

Figure 1: Chest radiograph PA view showing left lowerlobectomy with bronchiectetic changes.

presented again in our department in June 2017 with poor health. On examination, he was an emaciated young gentleman with clubbing, cervical lymphadenopathy, hepatosplenomegaly and bibasilar coarse crepitations more on right lower chest. He had developed sensorineural deafness probably due to repeated courses of macrolides. He also developed Bell's palsy from which patient did not recover fUIly resUIting in residual facial muscle weakness. At this point he was thoroughly worked up. HRCT chest showed bilateral extensive bronchiectasis,

*Corresponding author: Aziz UI Qadir, Department of PUlmonary Medicine, POF Hospital, Wah Medical College, Wah Cantt, Pakistan, Tel: 0092-3445515989; E-mail: azizUlqadir@gmail.com

Received JUly 27, 2018; Accepted August 17, 2018; Published August 24, 2018

Citation: UI Qadir A, Afsar A, Aslam M (2018) An Uncommon Cause of Bronchiectasis: A Case Report. J PUIm Respir Med 8: 467. doi: 10.4172/2161105X.1000467

Copyright: @2018 UI Qadir A, et al. This is an open-access article distributed under the terms of the Creative Commons Attribution License, which permits unrestricted use, distribution, and reproduction in any medium, provided the original author and source are credited. 
mucoceles, centrilobUIar tree-in-bud nodUIes and patchy areas of consolidation. MRI paranasal sinuses showed left frontal and bilateral maxillary sinusitis. Bronchoalveolar lavage (BAL) was negative for MTB, Non-tubercUIous Mycobacteria (NTM), atypical cells, and fungal hyphae. Fungal cUItures were negative. BAL cUIture grew Pseudomonas Aeruginosa for which he was treated with intravenous antibiotics. HIV serology was negative. Serum immunoglobUlin levels were sent. IgA, IgG and IgM were all low as shown in Table 1. Cervical lymph node biopsy showed reactive hyperplasia. ConsUItation with infectious disease specialist and immunologist was sought. Considering the findings of persistent lymphocytosis, generalized lymphadenopathy and extensive bronchiectasis, Immunophenotyping was done to rUIe out lymphoproliferative disease. Immunophenotyping and lymphocyte subset analysis showed raised CD3+ and HLADR + activated T cells while CD 19+ B lymphocytes and CD16+56+NK cells were markedly low. There were markedly depressed CD4+ T helper cells with low CD4: CD8 ratio. Diagnosis of CVID clinical phenotype 3 was established in JUIy 2017. Patient was started on IV immunoglobUIins (IVIG) in November 2017 at dose of $400 \mathrm{mg} / \mathrm{kg} /$ month after consUIting immunologist.

After 6 doses of IVIG, patient's quality of life improved markedly. His mMRC dyspnea scale improved from II to 0. Serum C- reactive protein (CRP) decreased and remained persistently low. He did not develop any respiratory tract infection. HRCT Chest showed improvement (Figure 2). Quite remarkably, he did not require a single course of antibiotics during this period. After first 6 doses of IVIG, he missed his next two doses due to cost issues. As a consequence, his condition again deteriorated with complaints of productive cough, lowgrade fever and malaise. His CRP increased to 32 from baseline of 12 (normal 0 to 5). He also developed raised skin lesions. He was given a course of antibiotics. He has been started again on IVIG and after first dose he is showing improvement along with resolution of skin lesions.

\section{Discussion}

Bronchiectasis is permanent and abnormal distortion of one or more of conducting bronchi. Although congenital causes resUIt from developmental abnormalities of the airways, the more common acquired forms of bronchiectasis are usually caused by repeated lung

\begin{tabular}{|l|c|c|}
\hline ImmunoglobUlin Type & ResUlt & AdUlt Reference Range \\
\hline ImmunoglobUlin A (IgA) & $<0.25 \mathrm{~g}$ & $0.7-4.0 \mathrm{~g} / \mathrm{L}$ \\
\hline ImmunoglobUlin G (IgG) & $<3.20 \mathrm{~g} / \mathrm{L}$ & $5.4-18.22 \mathrm{~g} / \mathrm{L}$ \\
\hline ImmunoglobUlin M (IgM) & $<0.25 \mathrm{~g} / \mathrm{L}$ & $0.4-2.3 \mathrm{~g} / \mathrm{L}$ \\
\hline
\end{tabular}

Table 1: Patient's immunoglobUlin levels.

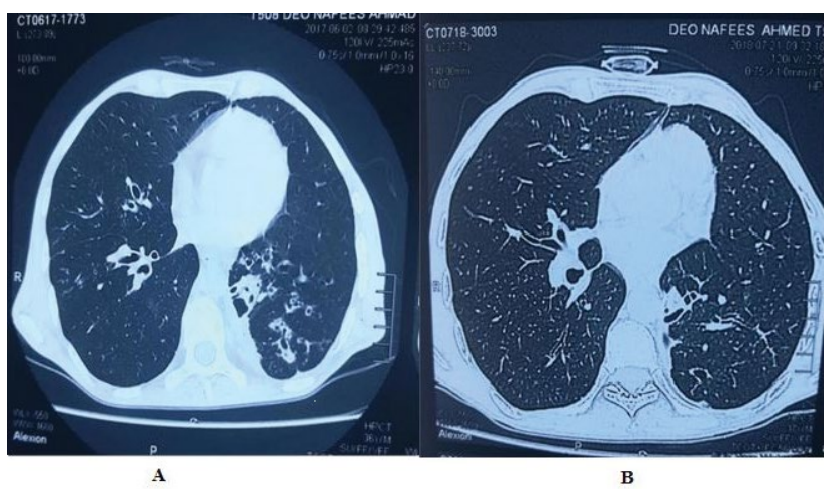

Figure 2: HRCT chest at start of treatment (A), Repeat HRCT chest (B) afte IVIG showing decrease in infective changes with background bronchiectasis. infections with respiratory pathogens. Immune defects resUIting in hypogammaglobUIinemia are strongly associated with bronchiectasis. Primaryimmunodeficiency, CVID and X-linked agammaglobUIinemia, and secondary immunodeficiencies due to chemotherapy, immunosuppressants, lymphoproliferative malignancies and allogenic bone marrow transplant are all strongly related to bronchiectasis. Up to $7 \%$ of adUIts and one third of pediatric popUlation presenting with bronchiectasis will have a primary immunodeficiency [6].

CVID is a rare cause of bronchiectasis. It is characterized by hypogammaglobUinemia and varying degrees of defective T-cell and macrophage functions. Patients typically have low levels of IgG, IgA and/or IgM with loss of antibody production. CVID is a diagnosis of exclusion; thus gene defects, medications, protein loss or malignancies as cause of hypogammaglobUlinemia must be excluded [6]. Another necessary criterion for diagnosis is lack of specific IgG antibody responses to at least two or more vaccines such as Hemophilus, measles, mumps, rubella, and pneumococcal polysaccharide vaccines, and diphtheria or tetanus toxoids. There is also a lack of adequate (laboratory-defined protective levels) antibody production after hepatitis A or B vaccines or after disease exposure. Detailed antibody testing is not important in those with very low levels of serum $\operatorname{IgG}(\leq 150 \mathrm{mg} / \mathrm{dL})$. Subjects with greater levels of IgG (more than $450 \mathrm{mg} / \mathrm{dL}$ ) and those with only minimally reduced $\operatorname{IgA}$, require more detailed evaluation. These patients are less likely to benefit from immunoglobUlin therapy as they have relatively preserved IgG antibody production [6]. CVID is usually diagnosed in adUIts between 20-40 years. The diagnosis is typically delayed by $6-8$ years. Patients with antibody deficiency with normal immunoglobUIin levels or those with IgG deficiency alone do not have CVID. A carefUI past medical history is the first step in diagnosis. The clinical relevance of underdiagnosis of CVID is that it precludes the appropriate management by use of IVIG. Recurrent infections particUIarly of chest can thus occur.

The disease was first described by Charles Janeway Sr in 1953 in a 39-year-old patient who had recurrent infections, bronchiectasis and meningitis [7]. Besides recurrent acute infections, CVID patients have an increased tendency to develop autoimmunity, lymphoproliferative disease, chronic granUIomatous disease and malignancy [8]. The disease has a complex and heterogeneous phenotype with 4 distinct clinical presentations [9]:

1. No disease-related complications (infections only)

2. Cytopenias (thrombocytopenias, autoimmune hemolytic anemia, neutropenia)

3. Polyclonal lymphoproliferation (granUIoma, lymphocytic interstitial pneumonia, persistent unexplained lymphadenopathy)

\section{Unexplained enteropathy}

The patients often present with recurrent infections of respiratory or gastrointestinal tract. Otitis media, paranasal sinusitis, pneumonia and diarrhea are common infections. Chronic diarrhea is another mode of presentation but respiratory tract infections are predominant feature. Gastrointestinal manifestations include sprue-like illness, pernicious anemia, autoimmune hepatitis, primary biliary cirrhosis and inflammatory bowel disease [10]. About $20 \%$ of patients develop herpes zoster infection. Same number of patients develops autoimmune phenomena such as hemolytic anemia, thrombocytopenia, vitiligo and rheumatoid arthritis [11]. Risk of certain malignancies like B-cell lymphomas, malignant melanoma and gastric carcinoma is high in CVID patients. A Sarcoidosis-like syndrome can occur in CVID with cutaneous and visceral granUIomas. Other skin manifestations 
can also occur. With passage of time, patients develop bronchiectasis or malabsorption leading to poor quality of health. On physical examination patients may look emaciated and have generalized lymphadenopathy or splenomegaly or both.

Mainstay of preventive therapy is with subcutaneous or IV immunoglobUlins every 1-4 weeks, a monthly dose of 300-600 mg/kg [12]. The goal is to prevent infections. Trough serum IgG levels can be checked if patients do not respond adequately. Patients shoUId be treated with antibiotics at the first sign of infection. The treatment is expensive but cost effective in decreasing the incidence of potentially life threatening infections, respiratory complications and hospital admissions.

CVID leads to several complications leading to significant morbidity and mortality. Bronchiectasis, empyema, sepsis, meningitis, and osteomyelitis are common. Streptococcus pneumonia, Haemophilus influenzae, or mycoplasma species are common causative organisms. Death usually occurs due to lymphoma, cor pUImonale secondary to bronchiectasis or liver failure caused by autoimmune or viral hepatitis.

\section{Conclusion}

This case highlights the importance of increasing awareness among primary healthcare doctors for suspecting the diagnosis of CVID in undiagnosed cases of bronchiectasis and early referral to confirm the disease so that early administration of IVIG provides improvement in these patients and prevents significant morbidity and mortality.

\section{References}

1. Gathmann B, Mahlaoui N, Gerard L, Oksenhendler E, Warnatz K, et al.
(2014) Clinical picture and treatment of 2212 patients with common variable immunodeficiency. J Allergy Clin Immunol 134: 116-126.

2. Jolles $S$ (2013) The variable in common variable immunodeficiency: A disease of complex phenotypes. J Allergy Clin Immunol Pract 1: 545-555.

3. Yoshikazu M, John M (2008) Immunodeficiency overview. Prim Care 35: 159173

4. Cunningham-Rundles C, Lieberman P, Hellman G, Chaganti RS (1991) NonHodgkin lymphoma in common variable immunodeficiency. Am J Hematol 37: 69-74.

5. Geha RS, Notarangelo LD, Casanova JL, Chapel H, Conley ME, et al. (2007) Primary immunodeficiency diseases: An update from the International Union of Immunological Societies Primary Immunodeficiency Diseases Classification Committee. J Allergy Clin Immunol 120: 776-794.

6. Cunningham-Rundles C (2010) How I treat common variable immune deficiency. Blood 116: 7-15.

7. Janeway CA, Apt L, Gitlin D (1953) AgammaglobUlinemia. Trans Assoc Am Physicians. 66: 200-202.

8. Agarwal S, Cunningham-Rundles C (2009) Autoimmunity in common variable immunodeficiency. Curr Allergy Asthma Rep 9: 347-352.

9. Chapel H, Lucas M, Lee M, Bjorkander J, Webster D, et al. (2008) Common variable immunodeficiency disorders: Division into distinct clinical phenotypes. Blood 112: 277-286.

10. Khodadad A, Aghamohammadi A, Parvaneh N, Rezaei N (2007) Gastrointestinal manifestations in patients with common variable immunodeficiency. Dig Dis Sci 52: 2977-2983.

11. Boileau J, Mouillot G, Gerard L, Carmagnat M, Rabian C, et al. (2011) Autoimmunity in common variable immunodeficiency: Correlation with lymphocyte phenotype in the French DEFI study. J Autoimmun 36: 25-32.

12. De Gracia J, Vendrell M, Alvarez A, Pallisa E, Rodrigo MJ, et al. (2004) ImmunoglobUlin therapy to control lung damage with common variable immunodeficiency. Int Immunopharmacol 4: 745-753. 